\title{
The Clinicopathological Factors Associated with Disease Progression in Luminal A Breast Cancer and Characteristics of Metastasis: A Retrospective Study from A Single Center in China
}

\author{
JINGMING YE ${ }^{1 *}$, WENJUN WANG ${ }^{2 *}$, LING XIN $^{1}$, SIONED OWEN ${ }^{3}$, LING XU $^{1}$, XUENING DUAN $^{1}$, \\ YUANJIA CHENG ${ }^{1}$, HONG ZHANG $^{4}$, SHUANG ZHANG ${ }^{4}$, TING LI $^{4}$ and YINHUA LIU ${ }^{1}$ \\ ${ }^{1}$ Breast Disease Center, Peking University First Hospital, Beijing, P.R. China; \\ ${ }^{2}$ General Surgery, Third People's Hospital of Datong City, Datong, P.R. China; \\ ${ }^{3}$ Cardiff China Medical Research Collaborative (CCMRC), School of Medicine, Cardiff University, Cardiff, U.K.; \\ ${ }^{4}$ Pathology, Peking University First Hospital, Beijing, P.R. China
}

\begin{abstract}
Background/Aim: This study investigated the clinicopathological factors associated with outcomes in patients with Luminal A breast cancer. Patients and Methods: Retrospective analysis of the association of clinicopathological factors and breast cancer outcome in 421 patients with newly-diagnosed Luminal-A breast cancer that were enrolled from January 2008 to December 2014. Clinicopathological data were analyzed to validate the relationship with disease-free survival (DFS) and overall survival (OS). Kaplan-Meier curves and log-rank tests were used to analyze the value of clinicopathological factors (tumor size, node status and lymphovascular invasion), and subsequent Cox regression analysis revealed significant prognostic factors. Results: With a median of 61 months follow-up, the 5-year DFS and 5-year OS rate were 98.3\% and $99.3 \%$. Cox multivariate regression analysis showed that clinical anatomic stage, tumor size, status of lymph nodes, lymphovascular invasion and systemic treatment are strong prognostic factors for clinical outcome in patients with Luminal-A breast cancer. Of all 413 patients with stage I-III breast cancer, 14 presented with metastasis (3.4\%) during the follow up. Bone $(6 / 14,42.9 \%)$ was the most common site
\end{abstract}

*These Authors contributed equally to this study.

Correspondence to: Yinhua Liu, Breast Disease Center, Peking University First Hospital, Beijing 100034, P.R. China. Tel: +86 83575053, Fax: +86 01066552566, e-mail: liuyinhua@ medmail.com.cn

Key Words: Breast cancer, molecular subtype, Luminal A, distant metastasis, prognostic factor. of metastasis followed by liver $(5 / 14,35.7 \%)$ and lung (4/14, $28.6 \%$ ). The median survival time after metastasis was 20.4 months. Of all the sites of distant metastasis, liver metastasis was the only factor that affected survival time after metastasis $\left(\chi^{2}=6.263, p=0.012\right)$. Conclusion: Patients with Luminal A breast cancer have excellent outcomes. Liver metastasis is an important factor compressing the survival time after distant metastasis presents.

Nowadays, the five most commonly diagnosed cancers among women in China are breast, lung and bronchus, stomach, colorectal, and esophageal. Breast cancer accounted for $15 \%$ of all newly diagnosed invasive cancer cases in China in 2015. Amongst Chinese women in the age group 30-59 years breast cancer is the most commonly diagnosed cancer and is the leading cause of cancer death in women younger than 45 years old (1). Studies have shown that the mortality trend of breast cancer has increased in Chinese women between 1990 and 2009 (2). Nearly 10 years have passed since 2009 and there have been fundamental changes in the understanding of breast cancer biology and treatment strategies. Disease free survival and overall survival of breast cancer patients have also changed.

Breast cancer is identified by molecular biomarkers and classified into four different subtypes: Luminal A, Luminal B, HER2 overexpression and Triple Negative breast cancer. Due to ER and PR positive, HER2 negative and low Ki67 expression, Luminal A breast cancer has lower recurrence risk and better prognosis than the other molecular subtypes. In order to explore factors related with local recurrence and metastasis, a retrospective study was conducted to analyze clinicopathological factors to predict disease progression of patients with Luminal A breast cancer. 


\section{Patients and Methods}

Between January 2008 and December 2014, patients with newly diagnosed Luminal A breast cancer at the Breast Disease Center, Peking University First Hospital were enrolled. All were diagnosed by core needle biopsy (CNB) prior to treatment. Patients were excluded if clinicopathological data or follow-up information were unavailable. A total of 421 patients were enrolled in this retrospective study. Patients' anatomic stages and prognostic stages were restaged by the American Joint Committee on Cancer (AJCC) 8 th edition breast cancer staging system.

Immunohistochemistry (IHC) and tumor grade of breast cancer. Immunohistochemical (IHC) examination was routinely performed on breast cancer specimens obtained from CNB or surgery. IHC testing was performed according to the American Society of Clinical Oncology/College of American Pathologists guidelines (3, 4). ERand PR-positive status was defined as there being at least $1 \%$ positive nuclei in the sample. HER2-positive status was defined as IHC 3+ or FISH over-expression. Ki67 was measured in terms of its nuclear staining, the percentage of Ki67 positive cells were calculated as the percentage of MIB-1-positive cells in a total of 1000 malignant cells observed at high-power magnification $(\times 400)$. The histologic grade was determined using the modified ScarffBloom-Richardson grading system (Nottingham Combined Histologic Grade).

Molecular subtypes of breast cancer. Luminal A breast cancer was defined as ER and/or PR positive, HER-2 negative, Ki-67 <14\% according to the St. Gallen Consensus, 2011(5).

Treatment of patients. All patients received surgery (mastectomy or breast conserving surgery) and/or chemotherapy (neoadjuvant or adjuvant) followed by endocrine therapy. Radiation therapy was administered to patients who received breast conserving surgery or who had lymph-node metastasis. Sentinel lymph node biopsy was used to evaluate status of axillary lymph nodes. Patients with positive sentinel lymph node, or any lymph node metastasis proved by CNB prior to surgery, received axillary lymph node dissection.

Follow-up. All patients enrolled in this study had complete follow up information. Disease free survival (DFS) was defined as the interval from surgery to disease recurrence, either loco-regional or distant metastasis, or the date of last follow-up without any evidence of recurrence. Overall survival (OS) was calculated from the date of diagnosis of breast cancer to death or final follow-up.

Statistical analysis. Statistical comparisons of clinical outcomes, cancer stage and other clinicopathological data were assessed with SPSS software version19.0. The Kaplan-Meier method, log-rank test and Cox multivariate regression analysis were used to evaluate the differences between the anatomic staging subgroup and prognostic staging subgroups. All of the statistical tests and $p$-values were twotailed. $p<0.05$ was considered statistically significant.

\section{Results}

Patients characteristics. From January 2008 to December 2014, 446 patients with Luminal A breast cancer were diagnosed at the Breast Disease Center, Peking University
First Hospital, which accounted for 20.54\% (446/2171) of all patients diagnosed with breast cancer at the center. Twenty five patients were excluded because the clinicopathological data or follow-up information were unavailable. A total of 421 patients were finally enrolled in this retrospective study. The median age of patients was 56 years old (24 to 91), with 8 cases (1.9\%) under 35 years old, 293 cases $(69.6 \%)$ between 35 to 65 , and 120 cases $(28.5 \%)$ older than 65 years old. Of all cases, 166 cases $(39.4 \%)$ were premenopausal women and $255(60.6 \%)$ were post-menopausal women. A total of 106 cases $(25.2 \%)$ underwent breast conserving surgery and radiation therapy while the other 315 cases underwent mastectomy. Fifty two cases (12.4\%) underwent neoadjuvant chemotherapy and surgery followed by endocrine therapy, 102 cases $(24.2 \%)$ underwent surgery followed by adjuvant chemotherapy and endocrine therapy, 267 cases $(63.4 \%)$ underwent surgery followed by endocrine therapy alone. Median follow-up time of this group of patients was 61 months ( 7 to 100 months). The rate of 5-year disease free survival was $98.3 \%$ and rate of 5-year overall survival (OS) was $99.3 \%$.

The clinicopathological characteristics of patients. Of all patients, 216 cases $(51.3 \%)$ were clinical stage I, 155 cases were clinical stage II $(36.8 \%), 42$ cases were clinical stage III $(10 \%)$ and 8 cases were clinical stage IV (1.9\%). For tumor size, 263 cases $(62.5 \%)$ were T1, 140 cases $(33.3 \%)$ were $\mathrm{T} 2,15$ cases $(3.6 \%)$ were $\mathrm{T} 3$ and 3 cases $(0.7 \%)$ were T4. For lymph node status, 284 cases $(67.5 \%)$ were negative, 100 cases $(23.8 \%)$ were N1, 29 cases $(6.9 \%)$ were N2 and 8 cases $(1.9 \%)$ were N3. Two hundred and ninety cases (68.9\%) were histopathological grade I, 124 cases grade II and 7 cases grade III. Four hundred and three cases $(95.7 \%)$ had no lymphovascular invasion whilst 18 cases $(4.3 \%)$ had lymphovascular invasion.

Correlation between clinicopathological characteristics and prognosis. Cox multivariate regression analysis showed that clinical stage (anatomic stage by AJCC cancer staging manual, 8th edition), tumor size, lymph nodes status, systemic treatment and lymphovascular invasion were factors related with clinical outcome of Luminal A breast cancer. Age, menopausal status, surgery and tumor grade were not related with disease progression. Clinicopathological characteristics of patients with Luminal A breast cancer which were associated with clinical outcome are listed in Table I.

The characteristics of patients with local recurrence and distant metastasis. Of the 413 patients with stage I-III breast cancer, 14 patients $(3.4 \%)$ presented with local recurrence or distant metastasis during follow-up, in which 2 cases (14.3\%) were pre-menopausal and 12 cases $(85.7 \%)$ were post-menopausal. The mean age of diagnosis amongst the 14 
Table I. Clinicopathological characteristics associated with the prognosis of the Luminal A breast cancer patients.

\begin{tabular}{|c|c|c|c|c|c|c|c|}
\hline & $\mathrm{N}(\%)$ & 5-year DFS (\%) & $\chi^{2}$ & $p$ & 5 -year OS (\%) & $\chi^{2}$ & $p$ \\
\hline AJCC anatomic stage & & & 11.933 & 0.003 & & 4.606 & 0.203 \\
\hline I & $216(51.3)$ & 99.4 & & & 98.9 & & \\
\hline II & $155(36.8)$ & 91.9 & & & 96.8 & & \\
\hline III & $42(10)$ & 97 & & & 94.6 & & \\
\hline IV & $8(1.9)$ & & & & 85.7 & & \\
\hline T-stage & & & 11.521 & 0.009 & & 23.626 & 0.000 \\
\hline $\mathrm{T} 1$ & $263(62.5)$ & 98.6 & & & 99.1 & & \\
\hline $\mathrm{T} 2$ & $140(33.3)$ & 92.1 & & & 95 & & \\
\hline $\mathrm{T} 3$ & $15(3.6)$ & 100 & & & 100 & & \\
\hline $\mathrm{T} 4$ & $3(0.7)$ & 100 & & & 50 & & \\
\hline Lymph node status & & & 20.532 & 0.000 & & 14.234 & 0.003 \\
\hline No & $284(67.5)$ & 99.2 & & & 97.9 & & \\
\hline $\mathrm{N} 1$ & $100(23.8)$ & 90.7 & & & 97.4 & & \\
\hline $\mathrm{N} 2$ & $29(6.9)$ & 100 & & & 100 & & \\
\hline N3 & $8(1.9)$ & 83.3 & & & 72.9 & & \\
\hline Lymphvascular invasion & & & 14.029 & 0.000 & & 5.957 & 0.015 \\
\hline No & $403(95.7 \%)$ & 97 & & & & & \\
\hline Yes & $18(4.3 \%)$ & 88.2 & & & & & \\
\hline System Treatment & & & 4.287 & 0.117 & & 6.179 & 0.046 \\
\hline $\mathrm{NAC}+\mathrm{ET}$ & $52(12.4)$ & 93.8 & & & 94 & & \\
\hline $\mathrm{AC}+\mathrm{ET}$ & $102(24.2)$ & 97 & & & 98 & & \\
\hline ET & $267(63.4)$ & 96.7 & & & 97.2 & & \\
\hline
\end{tabular}

NAC: Neoadjuvant chemotherapy; AC: adjuvant chemotherapy; ET: endocrine therapy.

Table II. The characteristics of patients with metastasis and their DFS and OS.

\begin{tabular}{|c|c|c|c|c|c|c|}
\hline Case no. & Anatomic stage & Prognostic stage & Patterns of metastasis & DFS (months) & OS (months) & Status \\
\hline 1 & IIA & IB & Lung metastasis & 71.9 & 92.7 & alive \\
\hline 2 & IIA & IB & Lung metastasis & 58.1 & 58.5 & dead \\
\hline 3 & IIB & IIB & Local recurrence & 46.3 & 50.1 & dead \\
\hline 4 & IIB & IB & Bone and liver metastasis & 45.5 & 75.1 & dead \\
\hline 5 & IA & IA & Bone metastasis & 33.9 & 88.7 & alive \\
\hline 6 & IIIC & IIIB & Liver metastasis & 34.6 & 38.3 & dead \\
\hline 7 & IIIC & IIIA & Liver metastasis & 83.1 & 87.6 & alive \\
\hline 8 & IIA & IB & Liver and Cecal metastasis & 31.7 & 36.2 & dead \\
\hline 9 & IIA & IB & Lung metastasis & 13.6 & 70.6 & alive \\
\hline 10 & IIA & IB & Bone metastasis & 4.0 & 66.1 & alive \\
\hline 11 & IIB & IB & Bone metastasis & 40.0 & 50.0 & alive \\
\hline 12 & IIA & IB & Local recurrence & 37.1 & 47.2 & alive \\
\hline 13 & IIB & IIB & Bone, liver, lung and pancreas metastasis & 10.0 & 27.2 & dead \\
\hline 14 & IIB & IIB & Bone metastasis & 6.0 & 32.2 & alive \\
\hline
\end{tabular}

patients was 65.5 years old (range $=43-84$ ), whilst the mean age of recurrence was 70.5 years old (range $=46-86$ ). The initial anatomic stage and prognostic stage as determined by the 8th AJCC breast cancer staging system of 14 patients are listed in Table II. Four patients had no differences between their anatomic and prognostic stage. The characteristics of metastasis and their clinical outcomes are also shown in
Table II. Two cases (14.3\%) developed local recurrence, whilst the other $12(85.7 \%)$ presented with distant metastasis. The most common site of distant metastasis was bone $(6 / 14$, $42.9 \%)$, followed by liver $(5 / 14,35.7 \%)$ and lung $(4 / 14$, $28.6 \%)$. Three cases $(21.4 \%)$ presented with multi-site metastasis when recurrence was diagnosed. For patients who presented with single metastatic sites 4 patients were 
Table III. The initial clinicopathological characteristics of patients with metastasis and their correlation with the survival time after metastasis.

\begin{tabular}{|c|c|c|c|c|}
\hline Characteristic & $\mathrm{N}(\%)$ & $\begin{array}{c}\text { 3-year survival } \\
\text { after metastasis } \\
(\%)\end{array}$ & $\chi^{2}$ & $p$-Value \\
\hline Age at diagnosis & & & 1.620 & 0.203 \\
\hline$\leq 65$ & $7(50.0 \%)$ & 71.4 & & \\
\hline$>65$ & $7(50.0 \%)$ & 25.0 & & \\
\hline Menstrual status & & & 2.729 & 0.520 \\
\hline Premenopausal & $2(14.3 \%)$ & 0.0 & & \\
\hline Postmenopausal & $12(85.7 \%)$ & 54.5 & & \\
\hline Breast Surgery & & & 0.022 & 0.881 \\
\hline $\mathrm{BCS}$ & $2(14.3 \%)$ & 50 & & \\
\hline Mastectomy & $12(85.7 \%)$ & 42.4 & & \\
\hline AJCC Stage & & & 12.666 & 0.002 \\
\hline I & $1(7.0 \%)$ & 100.0 & & \\
\hline II & $11(78.6 \%)$ & 42.4 & & \\
\hline III & $2(14.3 \%)$ & 0.0 & & \\
\hline Tumor size(mm) & & & 2.858 & 0.091 \\
\hline$\leq 20$ & $3(21.4 \%)$ & 100.0 & & \\
\hline$>20, \leq 50$ & $11(78.6 \%)$ & 25.0 & & \\
\hline Lymph node status & & & 12.000 & 0.002 \\
\hline No & $5(35.7 \%)$ & 60.0 & & \\
\hline N1 & $7(50.0 \%)$ & 35.7 & & \\
\hline $\mathrm{N} 2$ & $0(0.0 \%)$ & & & \\
\hline N3 & $1(7.1 \%)$ & 0.0 & & \\
\hline Tumor Grade & & & 1.742 & 0.187 \\
\hline I & $7(50.0 \%)$ & 62.5 & & \\
\hline II & $7(50.0 \%)$ & 42.9 & & \\
\hline III & $0(0.0 \%)$ & & & \\
\hline Systemic Treatment & & & 1.240 & 0.538 \\
\hline $\mathrm{NAC}+\mathrm{ET}$ & $7(50.0 \%)$ & 0.0 & & \\
\hline $\mathrm{AC}+\mathrm{ET}$ & $2(14.3 \%)$ & 50.0 & & \\
\hline ET alone & $5(35.7 \%)$ & 71.4 & & \\
\hline
\end{tabular}

BCS: Breast-conserving surgery; NAC: neoadjuvant chemotherapy, AC: adjuvant chemotherapy, ET: endocrine therapy.

diagnosed with bone metastasis $(28.6 \%), 3$ cases with lung metastasis (21.4\%) and 2 cases with liver metastasis $(14.3 \%)$.

The initial clinicopathological characteristics of patients with metastasis and their survival time after metastasis. The median survival time after metastasis diagnosed for the 14 Luminal A breast cancer patients was 20.4 (1 to 64) months. TNM stage and lymph node status were predictors of survival time after metastasis $\left(\chi^{2}=12.666\right.$ and $\chi^{2}=12.000$, $p=0.002$ ) (Table III). Age, menopausal status, tumor size, tumor grade, surgical and systemic therapy had no correlation with the survival time after metastasis.

Factors associated with survival time after metastasis in the patients with metastasis. Six of 14 patients were under 65 years old when they presented with metastasis; the other 8 patients
Table IV. The patterns and characteristics of metastasis and their correlation with survival time in patients with metastasis.

\begin{tabular}{lcccc}
\hline Characteristic & $\mathrm{N}(\%)$ & $\begin{array}{c}\text { 3-year survival } \\
\text { rate }(\%)\end{array}$ & $\chi^{2}$ & $p$-Value \\
\hline Age at recurrence & & & 0.756 & 0.385 \\
$\quad \leq 65$ & $6(42.8 \%)$ & 66.8 & & \\
$\quad>65$ & $8(57.2 \%)$ & 28.6 & & \\
DFS & $4(28.6 \%)$ & 75.0 & & 0.255 \\
$\quad \leq 24$ months & $10(71.4 \%)$ & 27.8 & & \\
$\quad>24$ months & & & 0.135 & 0.713 \\
Recurrent patterns & $2(14.3 \%)$ & 50.0 & & \\
$\quad$ Local & $12(85.7 \%)$ & 47.7 & & \\
$\quad$ Distant & & & & \\
Numbers of & & & & \\
recurrent sites & $11(78.6 \%)$ & 70 & & 0.102 \\
$\quad$ Single & $3(21.4 \%)$ & 0.0 & & \\
$\quad$ Multiple & & & 3.009 & 0.083 \\
Bone metastasis & $6(42.9 \%)$ & 55.6 & & \\
$\quad$ Yes & $8(57.1 \%)$ & 42.9 & & \\
$\quad$ No & & & 6.263 & 0.012 \\
Liver metastasis & $5(35.7 \%)$ & 0.0 & & \\
$\quad$ Yes & $9(64.3 \%)$ & 77.8 & & \\
$\quad$ No & & & 0.000 & 0.992 \\
Lung metastasis & $4(28.6 \%)$ & 50.0 & & \\
$\quad$ Yes & $10(71.4 \%)$ & 44.4 & & \\
$\quad$ No & & & & \\
\hline
\end{tabular}

were over 65 years old. Four patients who presented with metastasis did so within 24 months after treatment, whilst the other 10 presented over 24 months after treatment. We evaluated the correlation between factors such as age at recurrence, DFS, number of recurrent sites and metastasis patterns. There are statistical differences between patients with liver metastasis and without liver metastasis $\left(\chi^{2}=6.263\right.$, $p=0.012$ ), the other factors had no correlation with survival time after metastasis. One year survival rates of patients with liver, bone and lung metastasis were 50\%, 100\% and 75\% respectively. Three-year survival rates of patients with liver, bone and lung metastasis were $0 \%, 55.6 \%$ and $50 \%$ respectively. The Kaplan-Meier curve of patients with liver metastasis is shown in Figures 1-3. The 1-year and 3-year survival rate of patients with distant metastasis were $72.7 \%$ and $47.7 \%$ (Figure 4). One year and 3-year survival rates of patients with single site metastasis were $70 \%$ and $70 \%$ compared to patients with multi-site metastasis $(72.7 \%$ and $47.7 \%)$. The patterns and characteristics of metastasis related to survival time in patients with metastasis are showed in Table IV.

\section{Discussion}

Because of improvements in treatment and accurate identification of patients at risk, long term survival can be 

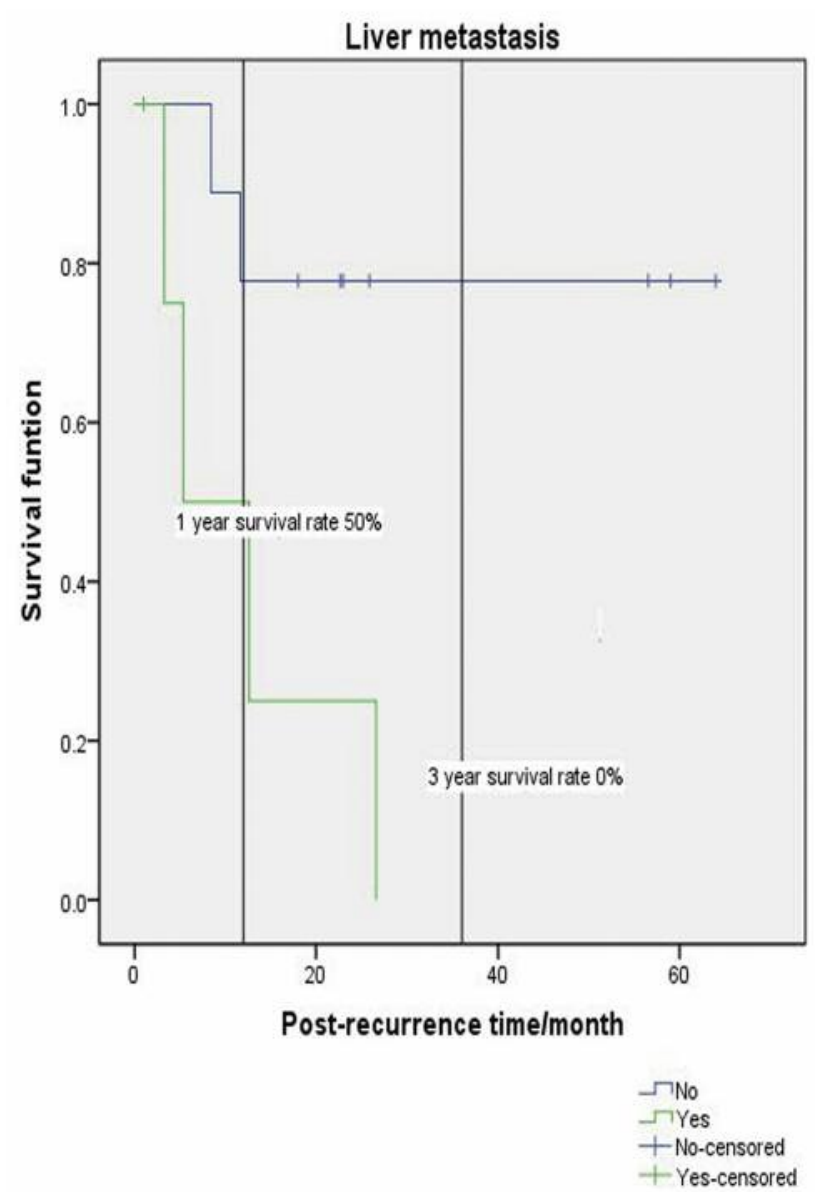

Figure 1. Survival time after metastasis for patients with liver metastasis. One-year survival rate of patients with liver metastasis was only 50\%, and no patients survived longer than 3 years. In contrast, three-year survival rate of patients without liver metastasis was $77.8 \%$ after metastasis were detected. There was a statistical difference between patients with liver metastasis and without liver metastasis in survival time after metastasis $\left(\chi^{2}=6.263, p=0.012\right)$.

achieved in breast cancer patients. Based on the expression of ER, PR, HER2 and Ki67, breast cancer is characterized by its molecular biomarkers and classified into four different subgroups: Luminal A, Luminal B, HER2 over-expression and Triple Negative breast cancer (6). Due to high risk of recurrence and poor prognosis, triple negative breast cancer has attracted a lot of attention in both clinical practice and research. Similarly, research has focused on the mechanism(s) and acquired treatment resistance of target therapies for HER2 positive breast cancer. Patients with hormone-receptor positive, HER2 negative and low expression of Ki67 breast cancer have favorable prognosis compared with other subtypes, and thus the pattern of

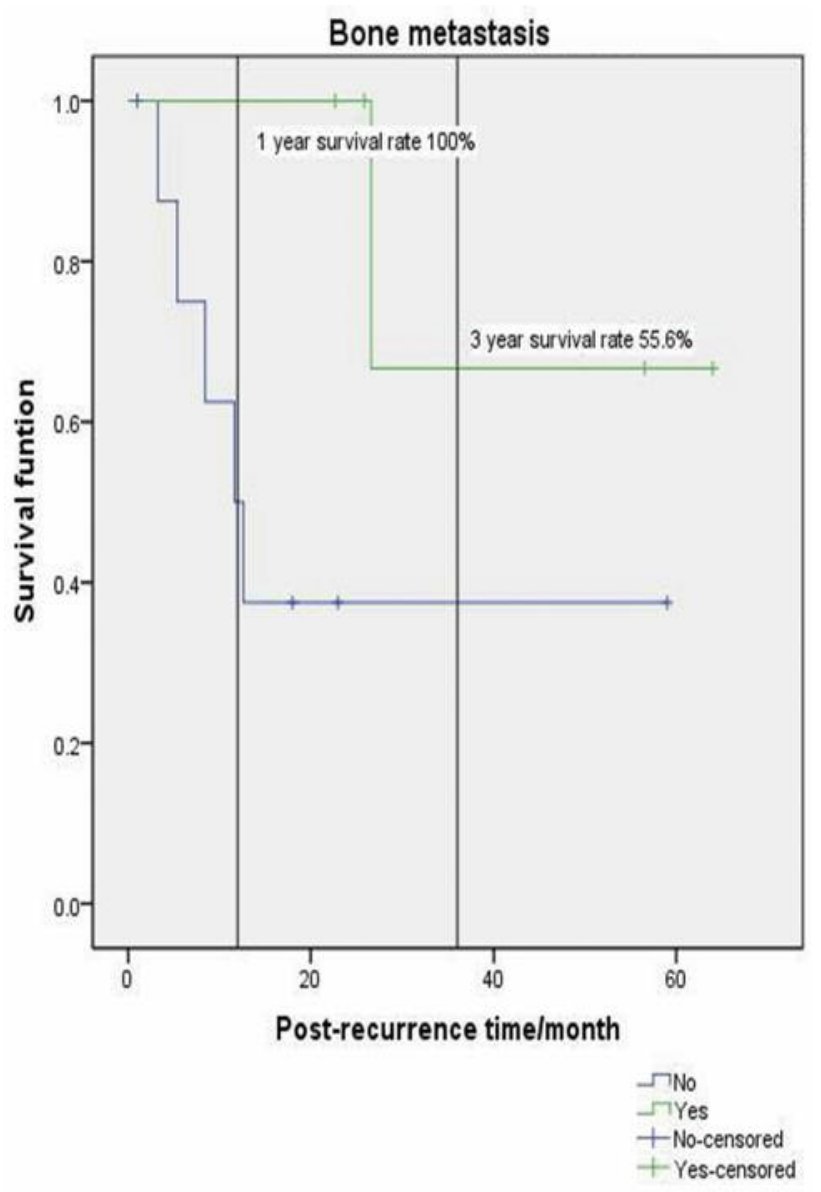

Figure 2. Survival time after metastasis for patients with bone metastasis. One year survival rates of patients with bone metastasis were $100 \%$. Three-year survival rates of patients with bone metastasis were $55.6 \%$. There was no statistical difference between patients with bone metastasis and without bone metastasis in survival time after metastasis $\left(\chi^{2}=3.009, p=0.083\right)$.

metastasis in this subgroup has not been paid much attention. Of all 421 Luminal A breast cancer patients enrolled in the analysis, the 5-year DFS was $98.3 \%$, and 5-year OS was $99.3 \%$. However, there were still 14 patients $(3.4 \%)$ who developed local recurrence or distant metastasis during follow-up, of which 4 patients presented with metastasis within 2 years after treatment. So it is essential to focus on the clinicopathological characteristics of Luminal A breast cancer and its patterns of metastasis, as well as to evaluate factors related with the survival time after metastasis. Thus we can identify patients of high recurrence risk who need escalated treatment to reduce the risk of metastasis and associated mortality. 




Figure 3. Survival time after metastasis for patients with lung metastasis. One-year survival rates of patients with lung metastasis were $75 \%$. Three-year survival rates of patients with lung metastasis were $50 \%$. There was no statistical difference between patients with lung metastasis and without lung metastasis in survival time after metastasis $\left(\chi^{2}=0.000, p=0.992\right)$.

We summarized the clinicopathological characteristics of patients and retrospectively analyzed their correlation with clinical outcomes of Luminal A breast cancer. Patients with Luminal A breast cancer accounted for $20.54 \%$ of all breast cancer patients during the same period in our center, consistent with $21.7 \%$ reported by Ahn et al. (7). Median age of the 421 patients with Luminal A breast cancer was 56 (24 to 91 ) years old, of which 293 patients $(69.6 \%)$ were between 35 to 65 years old, indicating that the age of diagnosis with breast cancer is relatively young in Chinese women, and the proportion of pre-menopausal patients is also relatively high (pre-menopause $39.4 \% \mathrm{vs}$. menopause $60.6 \%$ ). Clinical stage, tumor size, lymph node status, systemic treatment and lymphovascular invasion are factors

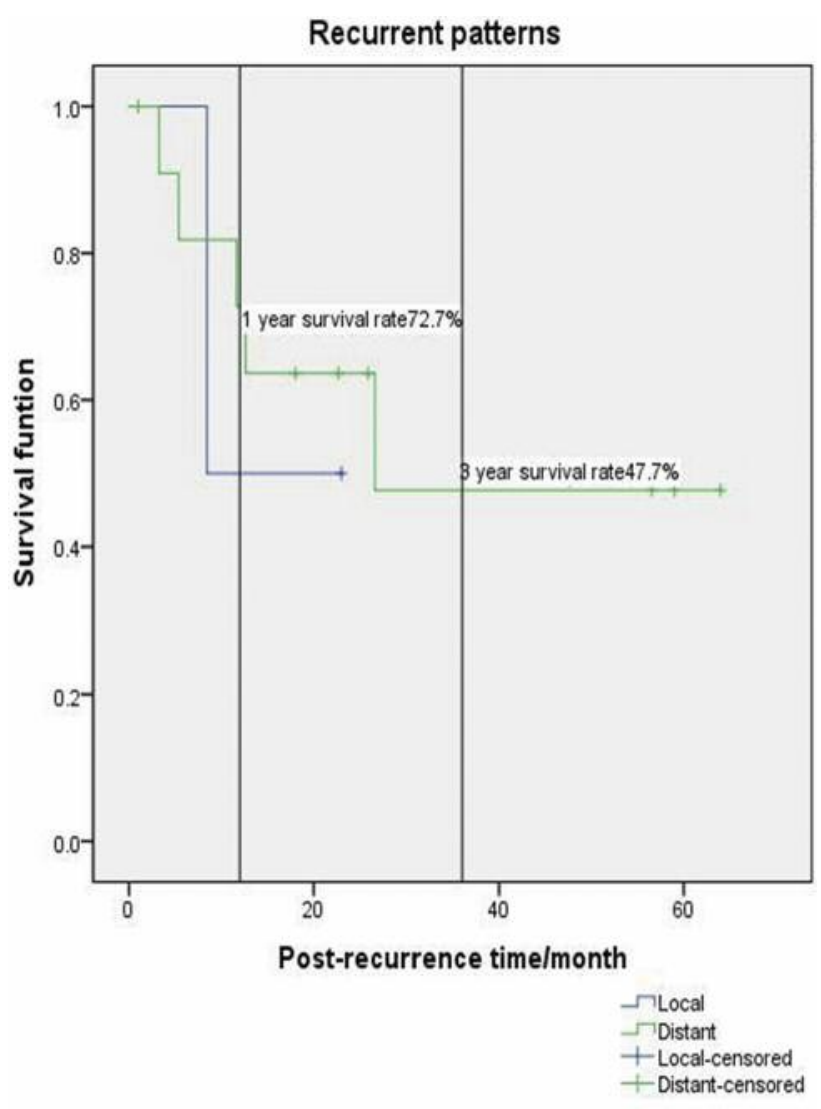

Figure 4. Survival time after metastasis for the patients with local recurrence and distant metastasis. Survival time after metastasis for the patients with local recurrence and distant metastasis. The one-year and three-year survival rate of patients with distant metastasis were $72.7 \%$ and $47.7 \%$. There was no statistical difference between patients with local recurrence and distant metastasis in survival time after metastasis $\left(\chi^{2}=0.135, p=0.713\right)$.

related with clinical outcome in patients with Luminal A breast cancer, whilst age, menstrual status, surgical procedure and tumor grade were not associated with prognosis. Despite 21-gene recurrence scores were not available, clinicopathological characteristics are still important predictive factors for the risk of metastasis in Luminal A breast cancer, and can be used to determine the need for adjuvant systemic therapy.

Few studies have discussed the clinicopathological characteristics of patients with luminal A breast cancer who presented with metastasis, and the impact of such characteristics on survival time after metastasis. There were 14 patients within the 413 patients of stage I-III Luminal A breast cancer patients who presented with metastasis in our 
study. We analyzed the clinicopathological characteristics at initial diagnosis and after detection of metastasis, in order to identify the clinical utility in predicting survival time after metastasis. As our study showed, there were 2 patients who presented with local recurrence and 12 patients who presented with distant metastasis, of which 3 (21.4\%) suffered from multi-site metastasis. The cases presenting bone, lung and liver metastasis alone were 4 (28.6\%), $3(21.4 \%)$ and $2(14.3 \%)$ respectively. The most common site of distant metastasis was bone $(6 / 14,42.9 \%)$, followed by liver $(5 / 14,35.7 \%)$ and lung $(4 / 14,28.6 \%)$. It is suggested that monitoring these organs as standard care during follow-up would allow for the detection of metastasis earlier. Although Luminal A breast cancer has favorable prognosis, patients who presented with metastasis suffered from a relatively short survival time, especially those with liver metastasis. The average survival time after metastasis was 20.4 months ( 1 to 64 months), but the 1year survival rate of patients with liver metastasis was only $50 \%$, and no one survived longer than 3 years. In contrast, the 3-year survival rate of patients without liver metastasis was $77.8 \%$ after metastasis was detected. There was a statistical difference between patients with liver metastasis and without liver metastasis in survival time after metastasis $\left(\chi^{2}=6.263, p=0.012\right)$.

Endocrine therapy is the preferred systemic therapy for Luminal A breast cancer, but for those who are at risk of local recurrence and distant metastasis, such as patients with a large tumor, positive lymph nodes, high tumor grade or lymphovascular invasion, adjuvant chemotherapy is still a necessary therapy to achieve better prognosis. Therefore, accurate evaluation of disease stages and recurrence risk is important to make therapeutic decisions. Netanely et al. (8) divided Luminal A breast cancer into two different prognostic groups using RNA-Seq and DNA methylation data. The analysis of these data provided two prognostic gene panels that dissect and explain tumor variability within Luminal A breast cancer which contributed to the advancement of subtype-specific diagnosis and treatment. However, limited evidence has confirmed the clinical usefulness of this assay. The AJCC 8th breast cancer staging system suggests multigene panel testing being routinely performed and acts as part of the prognostic staging system with biological markers including ER, PR, HER2, and tumor grade to provide more information about disease prognosis (9). The 21 -gene assay can predict patients who could benefit from adjuvant chemotherapy. Patients with low recurrence scores $(\mathrm{RS}<11)$ can safely avoid adjuvant chemotherapy, but adjuvant chemotherapy must be taken into consideration for those with high recurrence score $(\mathrm{RS}>25)$. Disparities in access and high cost make it difficult to maximize the clinical utility of multigene panels in China.
By adding important biological factors (HR status, HER2 status, tumor grade and gene panels) to TNM staging system, the AJCC 8th edition puts forward a breast cancer prognostic staging system, which differs from the original anatomic stages in some patients. In the 14 patients with Luminal A breast cancer who presented with metastasis, 10 of whom down staged according to prognostic staging system of AJCC 8th edition compared to their anatomic stages (Table II). These patients had received chemotherapy according to their original anatomic stages by the AJCC 7th edition cancer staging system, though it appears they didn't benefit from chemotherapy. With this new prognostic staging system, they might have had the chance to de-escalate chemotherapy so as to reduce unnecessary adverse effects.

Precise disease staging is the way to personalized treatment, especially in Luminal A breast cancer, which has a more favorable prognosis than any other subtype. The clinicopathological information such as tumor size, status of lymph nodes are still the basis of the breast cancer staging system, together with biological markers and multigene panels, we can reveal more about the extrinsic and intrinsic characteristics of tumors and make treatments more personalized.

\section{Conflicts of Interest}

The Authors issued no conflicts of interest.

\section{Acknowledgements}

This study was supported by research grants from the Beijing Municipal Commission of Health and Family Planning (No. 20091100), the Beijing Municipal Science and Technology Commission (No. D090507043409010 and Z131107002213007) and from Precision Medicine Special Project of National Key Research and Development Program (2016YFC0901302), P.R.China.

\section{References}

1 Chen W, Zheng R, Baade PD, Zhang S, Zeng H, Bray F, Jemal A, Yu XQ and He J: Cancer statistics in China 2015. CA Cancer $\mathrm{J}$ Clin 66: 115-132, 2016.

2 Li C, Yu C and Wang P: An age-period-cohort analysis of female breast cancer mortality from 1990-2009 in China. Int J Equity Health 14: 76, 2015.

3 Hammond ME, Hayes DF, Dowsett M, Allred DC, Hagerty KL, Badve S, Fitzgibbons PL, Francis G, Goldstein NS, Hayes M, Hicks DG, Lester S, Love R, Mangu PB, McShane L, Miller K, Osborne CK, Paik S, Perlmutter J, Rhodes A, Sasano H, Schwartz JN, Sweep FC, Taube S, Torlakovic EE, Valenstein P, Viale G, Visscher D, Wheeler T, Williams RB, Wittliff JL and Wolff AC: American Society of Clinical Oncology/College Of American Pathologists guideline recommendations for immunohistochemical testing of estrogen and progesterone receptors in breast cancer. J Clin Oncol 28 : 2784-2795, 2010. 
4 Wolff AC, Hammond ME, Hicks DG, Dowsett M, McShane LM, Allison KH, Allred DC, Bartlett JM, Bilous M, Fitzgibbons P, Hanna W, Jenkins RB, Mangu PB, Paik S, Perez EA, Press MF, Spears PA, Vance GH, Viale $G$ and Hayes DF: Recommendations for human epidermal growth factor receptor 2 testing in breast cancer: American Society of Clinical Oncology/College of American Pathologists clinical practice guideline update. J Clin Oncol 31: 3997-4013, 2013.

5 Goldhirsch A, Wood WC, Coates AS, Gelber RD, Thürlimann B, Senn HJ and Panel members: Strategies for subtypes dealing with the diversity of breast cancer: highlights of the St. Gallen International Expert Consensus on the Primary Therapy of Early Breast Cancer 2011. Ann Oncol 22: 1736-1747, 2011.

6 Esposito A, Criscitiello C and Curigliano G: Highlights from the 14(th) St Gallen International Breast Cancer Conference 2015 in Vienna: Dealing with classification, prognostication, and prediction refinement to personalize the treatment of patients with early breast cancer. Ecancermedicalscience 31: 1736-1747, 2015.

7 Ahn HJ, Jung SJ, Kim TH, Oh MK and Yoon HK: Differences in clinical outcomes between Luminal A and B Type breast cancers according to the St. Gallen Consensus 2013. Breast Cancer 18: 149-159, 2015.

8 Netanely D, Avraham A, Ben-Baruch A, Evron E and Shamir R: Expression and methylation patterns partition luminal-A breast tumors into distinct prognostic subgroups. Breast Cancer Res 18: $74,2016$.
9 The American Joint Committee on Cancer: AJCC Cancer Staging Manual. Amin MB, Edge S, Greene F, Byrd, DR, Brookland RK, Washington MK, Gershenwald JE, Compton CC, Hess KR, Sullivan DC, Jessup JM, Brierley JD, Gaspar LE, Schilsky RL, Balch CM, Winchester DP, Asare EA, Madera M, Gress DM, Meyer LR (eds.), Springer Publ Corp., New York, 2016.

10 Sparano JA, Gray RJ, Makower DF, Pritchard KI, Albain KS, Hayes DF, Geyer CE Jr., Dees EC, Perez EA, Olson JA Jr., Zujewski J, Lively T, Badve SS, Saphner TJ, Wagner LI, Whelan TJ, Ellis MJ, Paik S, Wood WC, Ravdin P, Keane MM, Gomez Moreno HL, Reddy PS, Goggins TF, Mayer IA, Brufsky AM, Toppmeyer DL, Kaklamani VG, Atkins JN, Berenberg JL and Sledge GW: Prospective Validation of a 21-Gene Expression Assay in Breast Cancer. N Engl J Med 373: 2005-2014, 2015.

11 NCCN Guidelines from the NCCN home page: https: //www.nccn.org/ professionals/physician_gls /pdf/breast.pdf

12 Zhou B, Ji K, Xin L, Zhang H, Xu L, Li T and Liu YH: Updates and interpretations of the 8th edition of AJCC breast cancer staging system. Chin J Prac Surg 37: 10-14, 2017.

Received May 2, 2017

Revised May 18, 2017

Accepted May 19, 2017 